Article

\title{
Devices for Continuous Recording of "Integral ECG" for Determining Vital Physiological Indicators of a Person's State and Their use in Bio Cybernetic Complexes
}

\author{
Bogatyrev Evgeniy ${ }^{1}$, Kodkin Vladimir ${ }^{2}$ \\ 1 South Ural State University, Chelyabinsk, Russia; evgeniy182@mail.ru \\ 2 South Ural State University, Chelyabinsk, Russia; kodkina2@mail.ru
}

Abstract: One of the rapidly developing research areas is the creation of systems. which are commonly referred to as cyberphysical complexes. In such systems, devices and complexes interact with a completely different physical nature. The role of a person in such systems usually consists in the formation of final tasks for "artificial intelligence" and executive mechanisms. The functioning of actuators is controlled by accurate information systems.

To monitor the human condition, analogues of medical diagnostic devices, which have completely different tasks, are most often used. At the same time, medicine in recent years has been very "personalized", which seriously brings a person closer to the "cyberphysical system." Equipment is rapidly developing in which a person becomes a full-fledged "participant" in the functioning of the complex. Examples are electric vehicles. Intelligent simulators and exoskeletons - in all such complexes, accurate and quick information about the physical state of a person is needed.

For these purposes, the most acceptable system for registering a person's electro-biopotentials is his ECG. This is one of the most important physiological functions. In addition, the ECG is the initial information for other important functional coordinates - for example, heart rate.

The paper presents the results of the development of software and hardware for ECG recording, which provide accurate ECG measurement from specially designed electrodes that have a large surface for reliable signal pick-up during movements and physical stress experienced by a person. The prospects of using these registration systems are shown.

Keywords: personalized medicine; "cyberphysical system; biocybernetic complexes; electrobiopotentials; ECG measurement; the quality of registration of an integral ECG

\section{Introduction}

The monitoring of vital physiological indicators of a person's state until now is a task that has been solved by medicine: its methods, equipment and specialists - doctors and nurses. During such control, the subjects are protected from external influences.

Resting is a prerequisite for serious medical examinations. The task of most of these examinations is to identify pathological changes in the functioning of the human body or the prerequisites for such.

However, in recent years, the quantity and quality of such tasks has changed significantly.

An example of other tasks can be monitoring the state of very healthy people, who, due to their occupation, have to make physical and nervous "super-efforts" - athletes, military men, rescuers. Monitoring systems for their condition should detect overvoltage, fatigue, critical stress. This is very different from monitoring the condition of sick people. At the same time, the control systems should 
work for a long time, without the participation of doctors and not interfere with the professional activities of the subjects.

The rapidly developing "personalized medicine" is solving similar problems. In this medicine, a person, diagnostic devices, computer tools for processing and transmitting information work together. Examples of such systems can be complexes with small-sized portable drug dispensers, the most common of which is insulin pumps for the treatment of patients with diabetes mellitus.

In modern science, complex systems combining devices and units that are very different in nature and modes of functioning are usually referred to as cyber-physical complexes - CPC[3-5]. In such systems, devices and complexes with completely different physical nature are united by a common goal. Very often such complexes interact with a person.

There is every reason to classify cyber-physical complexes with a person as "Bio Cybernetic Systems" (BCS), in which a person with his specific characteristics and physiology is the most important and integral "part". It should be noted that in KLF the functioning of executive mechanisms and artificial intelligence is controlled by information systems, the accuracy of which is very high [11]. The same cannot be said about controlling a person's condition.

In recent years, communication tools have developed so rapidly that many authors consider it a fait accompli that the technical solutions that will make a breakthrough in this direction implantable chips, non-contact blood spectrum analysis tools, controlled drug dispensers, etc. - all these and other technical solutions will solve many problems at a whole new level. But as the events of the year 20 showed, this "new time" for the majority of the world's population is still in the future. Even the most complex systems that can be classified as BCS - for example, modern insulin pumps, which are often called "artificial pancreas", require frequent and complex participation in human work. Moreover, the accuracy of such systems is low, and information about a person's condition is limited exclusively by the level of blood sugar with a rather large error. These BCS are clearly having problems with algorithm optimization.

In this regard, it seems a promising solution to the problem - the use of well-proven, over the course of many years, diagnostic information about the human condition. Such diagnostics should be recognized as electrocardiography (ECG). ECG is one of the most important physiological functions of a person. They measure the ECG for a long time, simply, accurately - from the arms, legs, from the esophagus, from the back, even though clothing[1,5-7]. However, in "serious" cardiology they are very skeptical about all ECGs, except for the one that is recorded by standard methods and from strictly defined areas on the surface of the human body. A huge amount of diagnostic materials and electrocardiography technologies most often relate only to the ECG recorded precisely by standard methods. For all other ECGs, cardiologists can only use R-Rmeasured heart rate - Heart rate (HR)[8-10].

\section{Formulation of the problem}

Registering a signal in fractions of a millivolt, even in the presence of interference, is not too difficult for modern electronics. The problems are rather the means of "picking up" signals[2]. Most often in recent years in the "traditional" ECG used disposable metallized self-adhesive electrodes. In addition to their merits, they also have some "complex" features. This is a small area and an absolute inability to install them on the human body by a non-specialist. To get around this problem, attempts have been made to build a "non-contact ECG" system[12-15]. Since the ECG is an alternating electrical signal with a spectrum from 0 to $150 \mathrm{~Hz}$, it is quite possible to catch it with modern amplifiers. But stability is needed, which non-contact ECG is no different. The use of Epic capacitive electrodes raises several questions. Firstly, capacitive sensors "catch" interference, secondly, they distort the classical ECG, and thirdly, they are connected in series to a person's clothing. they cannot increase the electrical capacitance formed by this garment because the capacities connected in series do not add up their values. The natural direction of improving the "removal" of ECG - increasing the area of the electrodes. At the same time, the possibility of obtaining differentiated chest leads (V1-V6) will be lost, but the reliability and stability of 
registration should appear during human movements. Such electrocardiography "removing" electrical signals from a relatively large surface of the skin $(30-100 \mathrm{sq} \mathrm{cm})$ can be called "Integral ECG".

\section{Research methods}

To solve the problem of reliable long-term ECG recording at South Ural State University, measuring electrodes made of conductive fabric fixed on the surface of a base made of a material with a shape memory effect were developed, while the base made of a material with a shape memory effect is convex from the side of fixing the conductive tissue, the thickness "Conductive layer" is 1-5 mm. This design provides a large area of contact of such electrodes with the human body, allows you to remove the ECG signal without interference, which are usually present in the recorded signal when a metal electrode of a large area - more than 30-50 sq cm - comes into contact with the human body. ECG signal. Electrodes made of conductive fabric were installed in clothes ("ECG - T-shirts"), in chairs and special belts (Fig. 1)[8-10]:

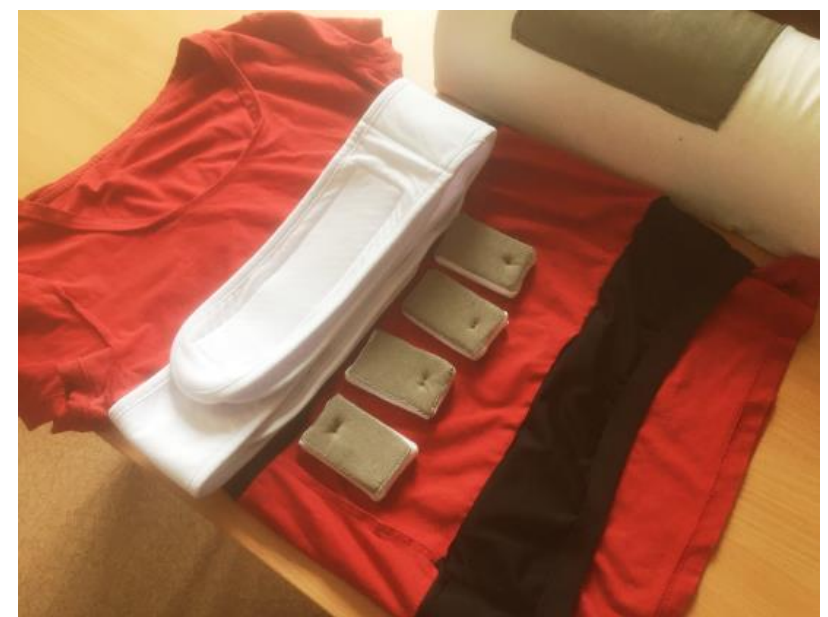

(a)

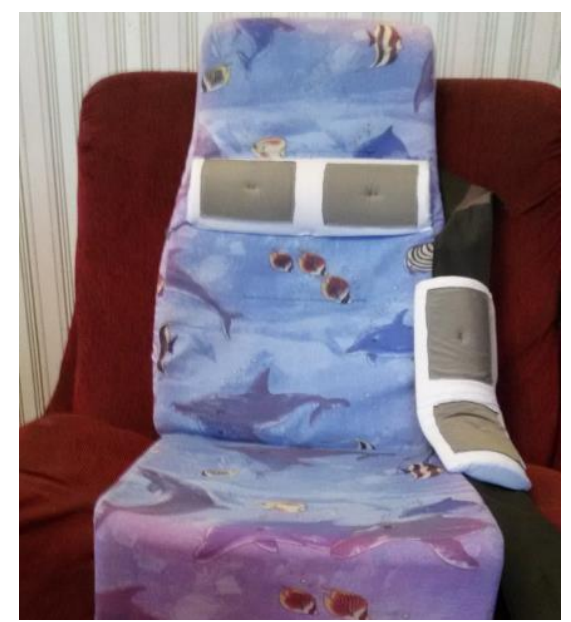

(b)

Figure 1. Electrodes made of conductive fabric embedded in clothes (a), in chairs (b)

In Fig. 2 shows a block diagram of a typical ECG "pickup" device[2]. For its reliable and accurate operation, it is necessary that all electrodes are installed exactly in their places on the human body. 


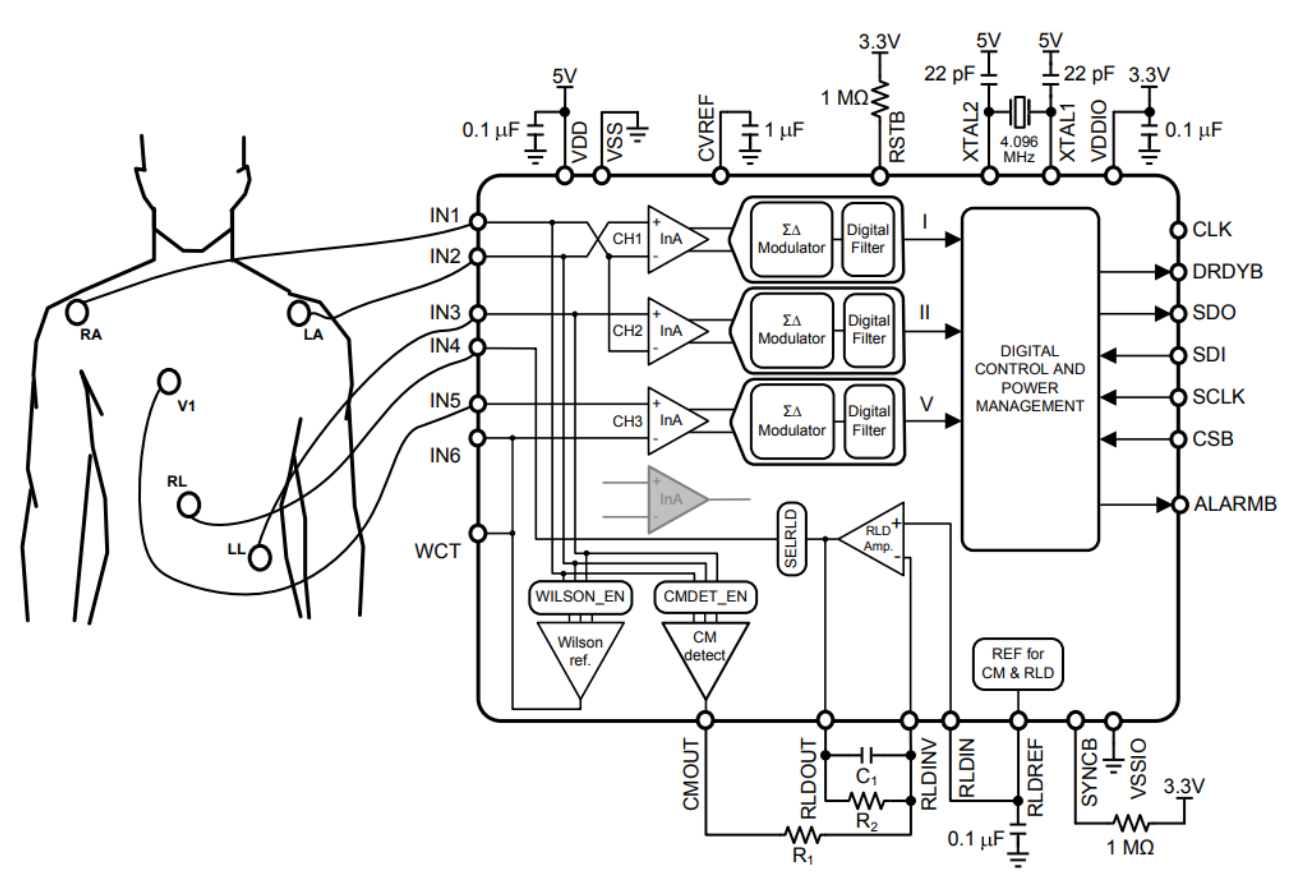

Figure 2. 5-Lead ECG Application

On the basis of a specialized device ADS1293, which implements this scheme, a recorder was developed with wireless signal transmission to a tablet for visualization and ECG analysis. Several experiments were carried out, the purpose of which was to establish whether it is possible, by combining a standard ECG recorder with large-area textile electrodes that "record" an integral ECG, to improve the reliability of ECG recording during human movements and whether the information obtained will be sufficient to conclude about his condition.

Textile electrodes were "sewn" into the T-shirt in places close to those intended for the microcircuit (Fig. 2)[1,5-7]. For the reliability of ECG recording during movements, the electrodes were combined according to the principle of "right" and "left" - that is, 5 electrodes were connected into groups "left" (2 electrodes) and "right" (3 electrodes). The experiments brought the following results:

Experimental research 1: The athlete wearing this shirt did a variety of physical exercises. Strength exercises, arm movements and lifting weights led to a change in the amplitude and even the sign of the R-wave, but did not cause serious movements of the ECG isoline. ECG diagrams in Fig. 3. The isoline is quite stable, although the amplitude of the cardiocomplexes varies from 2 to $0.2 \mathrm{mV}$ (points 1, 2, 3, - cardiocomplexes with different R-teeth).

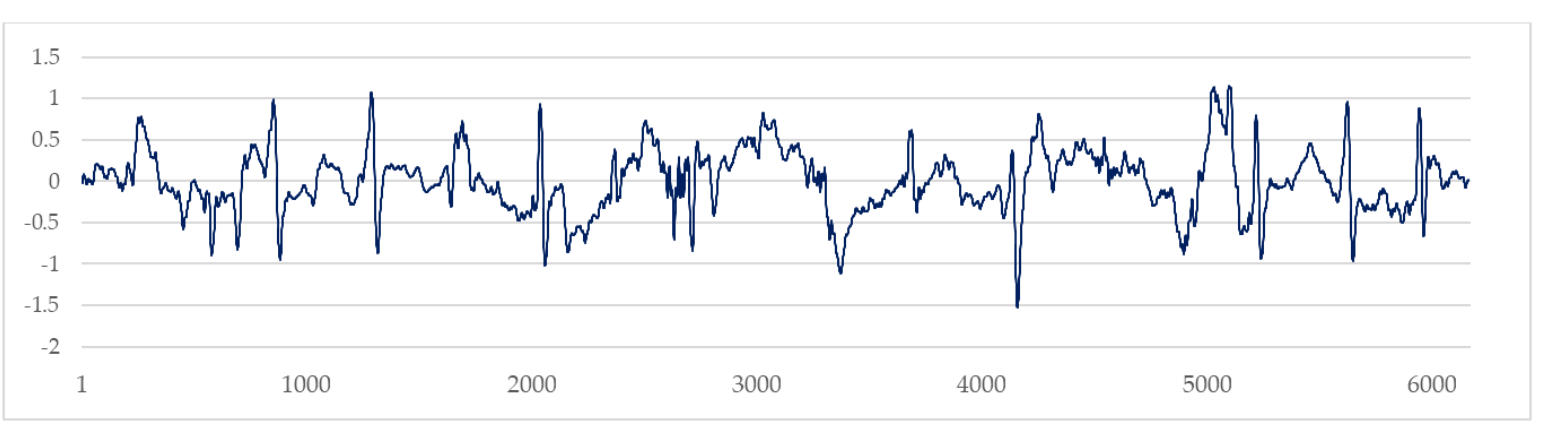

Figure 3. EKG of an athlete in an EKG - T-shirt

Figures 4 and 5 show the ECG of the same athlete while jogging. According to the signals in Fig. 4 , you can easily measure the R-R intervals and heart rate, and according to the amplified ECG signals in Fig. 5 - the intervals PQ, RS, QT, and the R, P, S, T waves. 


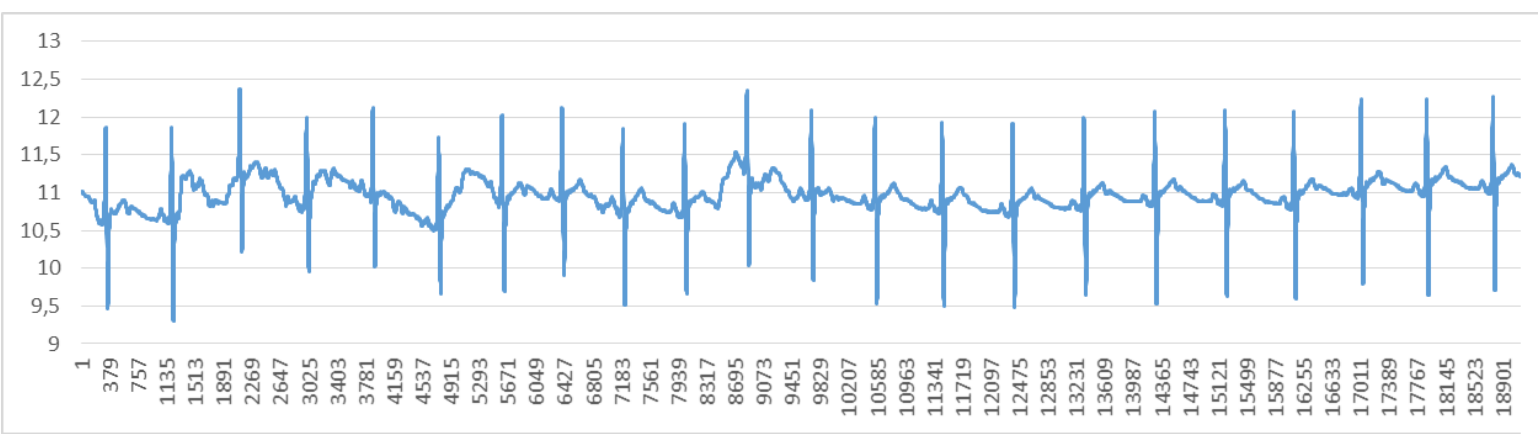

Figure 4. EKG of an athlete while jogging

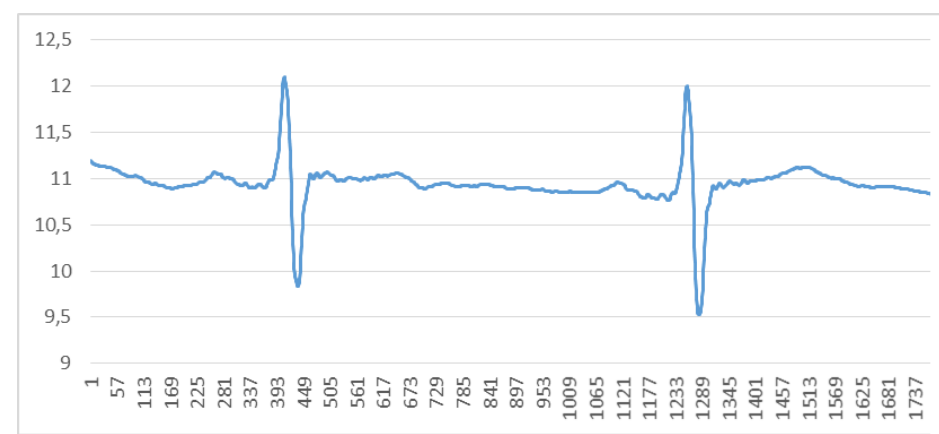

Figure 5. Enhanced ECG signals

Figures 6 and 7 show the ECG of an athlete during body movements - push-ups and bends. In this case, you can observe the jumps of the isoline by $30-35 \mathrm{mV}$. Whereas the amplitudes of the cardio complexes themselves remain 2-2.5 $\mathrm{mV}$. (points-1, 2, 3, 4) When increasing the scale of the ECG - Fig. 7, the elements of the ECG differ.

Thus, the "integral ECG" identifies not only the ECG and heart rate, but also the nature of human movements.

It should be noted that in these experiments the ECG recorder did not have significant low-pass filters for filtering the isoline movements with which Holter devices are equipped.

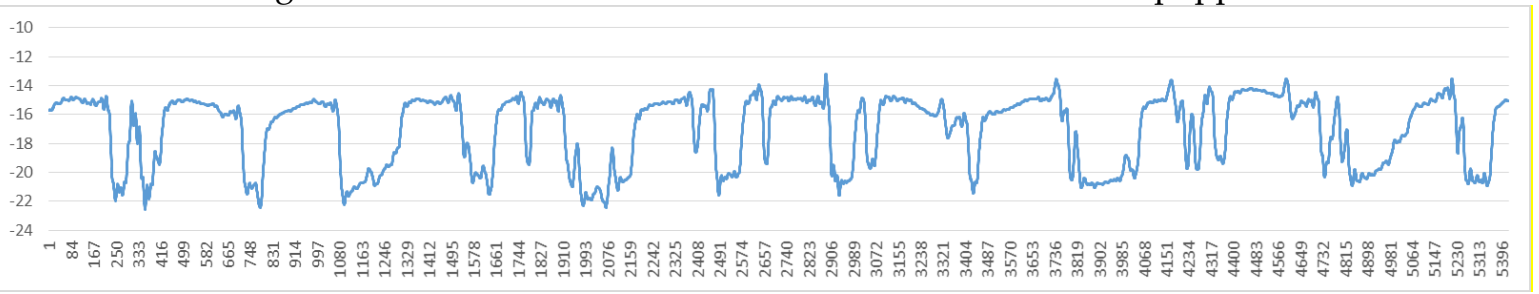

Figure 6. ECG of an athlete during body movements

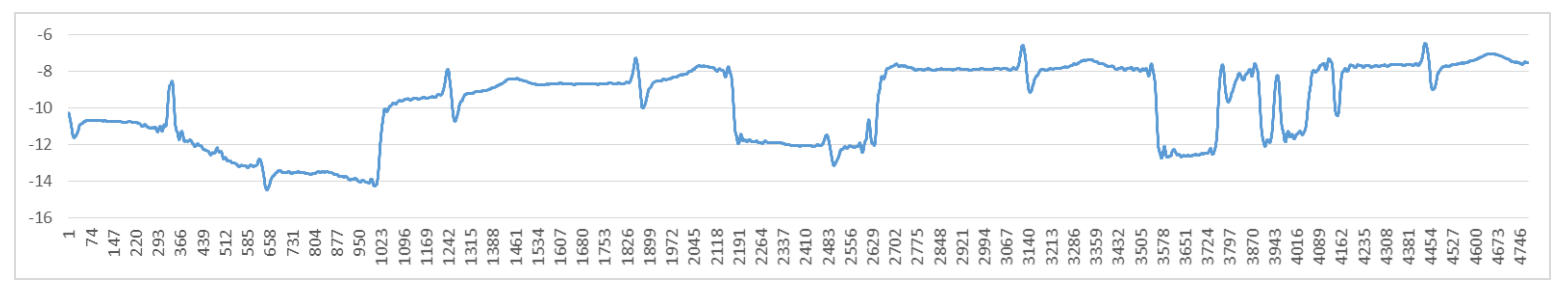

Figure 7. Enhanced ECG signals

During the tests, it was found that in the first few minutes of the athlete's performance of the exercises, there was no reliable contact of the electrodes with the skin surface. Subsequently, with sudden movements, contact was also broken.

Reliable contact and signal appeared 3-4 minutes after the start of the athlete's movements, i.e. most likely after sweat appeared on his skin. To test the influence of electrical active resistances in the electrical circuits of the electrodes, laboratory studies were carried out. A sinusoidal voltage 
with a frequency of $1 \mathrm{~Hz}$ and an amplitude of $6 \mathrm{mV}$ was connected to the input of the recorder (Fig. 8). Fig. 8a shows the signal when the signal is directly connected to the inputs of the recorder.

Additional active resistances, simulating a layer of insulation (clothing), were introduced into the measuring circuits of the recorder (Figure 8b). This led to increased interference. After increasing the additional resistances in the measuring circuits to $5 \mathrm{mOhm}$, the noise level of the reproduced signal increased to unacceptable (Fig. 8b). Moreover, connecting the compensating electrode to the source of the measured signal directly (without a resistor) did not reduce the noise. (Fig. 8c).

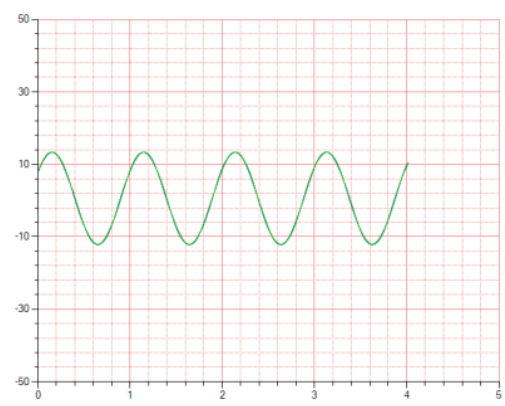

(a)

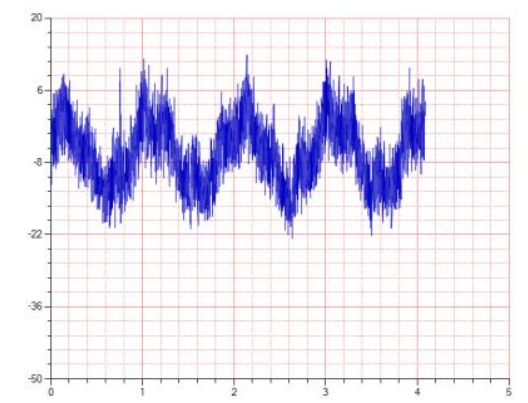

(b)

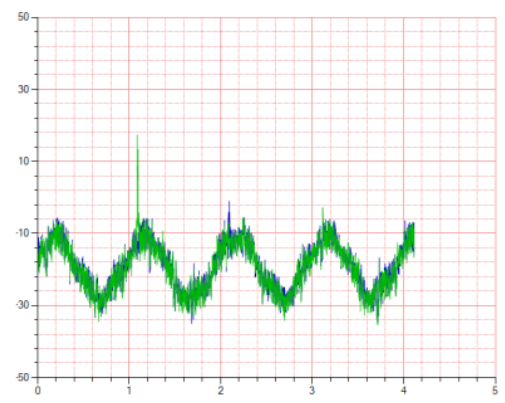

(c)

Figure 8. Influence of electrical active resistances in electrical circuits of electrodes

Thus, an ECG recorder with textile electrodes of a large area registers an "integral" ECG that is resistant to human movements and even capable of identifying the characteristic of movements, but the standard scheme for switching on the recorder's operational amplifiers is sensitive to active resistances in the leads and the compensating ECG channel, which makes the registration unstable.

Such properties of the recorder cannot be considered ideal for fulfilling the purposes of continuous ECG recording devices.

Experimental research 2: Another high-precision electrical signal recorder was used - a universal precision recorder of three independent electrical potentials with their subsequent digitization and computer processing. The diagram of one of the channels is shown in Fig. 9. The differences between the recorder circuits are in the connection of the compensating electrode. The compensating electrode of the second version of the ECG recorder is connected to the inverse input of the amplifier of the measured electrical signal. As you know, the inverse input of precision amplifiers has a very low input resistance. In this case, one of the poles of the source of the measured electrical signal (in this case, the ECG) is connected to the non-inverted input of the same amplifier. The conversion factor of the amplifier at the non-inverted input does not depend on the resistance at the non-inverted input. It can be anything, even very large, even insulation or clothing. In a standard recorder (first version), this circuit is exactly the same, only the compensating electrode is connected to the output of the operational amplifier. compensating bias, and the amplifier output is a point with high electrical potential. Such inclusion of the compensating electrode "breaks" the measured electrical potential and the operational transducer when the resistance at the noninverted input increases. The signal is measured by the recorder, but together with strong interference, behind which the ECG signal is lost. In the second variant, the interference will be seriously weakened, if only the compensating electrode is connected to the human skin, to almost any part of the body and the ECG can be observed when the significant electrodes are installed on the human body through a layer of tissue (clothing). 


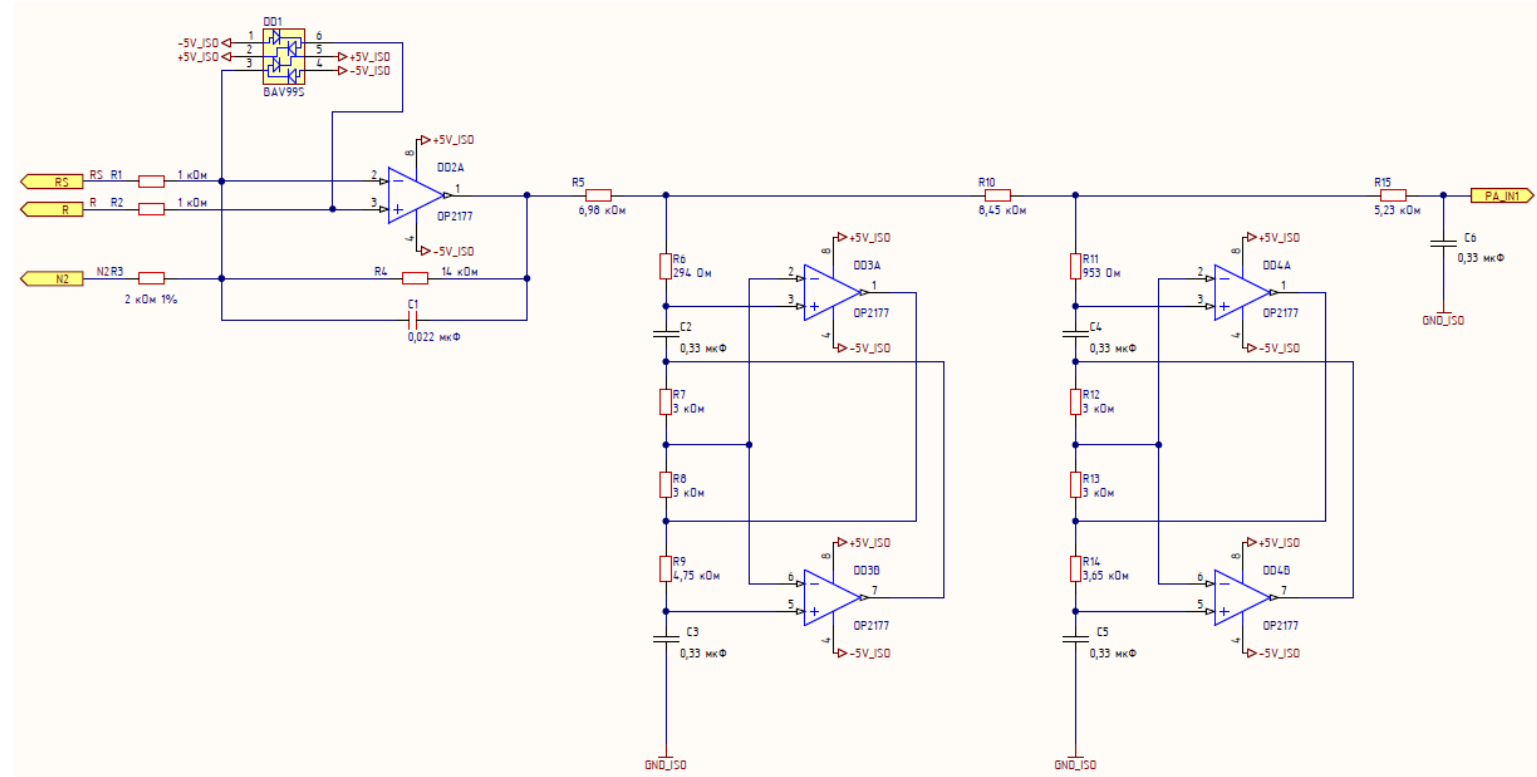

Figure 9. ECG derivation channel signal matching circuit

Tests have fully confirmed these provisions. Figure 10 shows the ECG diagrams recorded by the "second" recorder when the significant electrodes are installed on the clothes, and the compensating one on the human skin.

When the compensating electrode was disconnected from the human skin, the ECG signals were lost in interference. In Fig. 10, the moment of time "140.5 s" is the disconnection of the compensating electrode, In Fig. 11, the moment of time "160 s" is the connection of the same electrode to the skin of the subject.

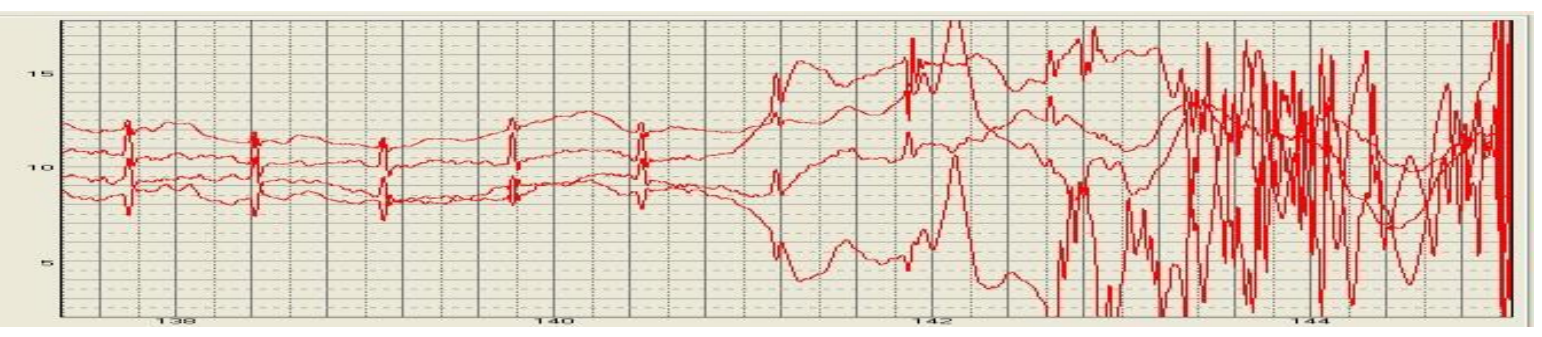

Figure 10. ECG with the disconnection of the compensating electrode

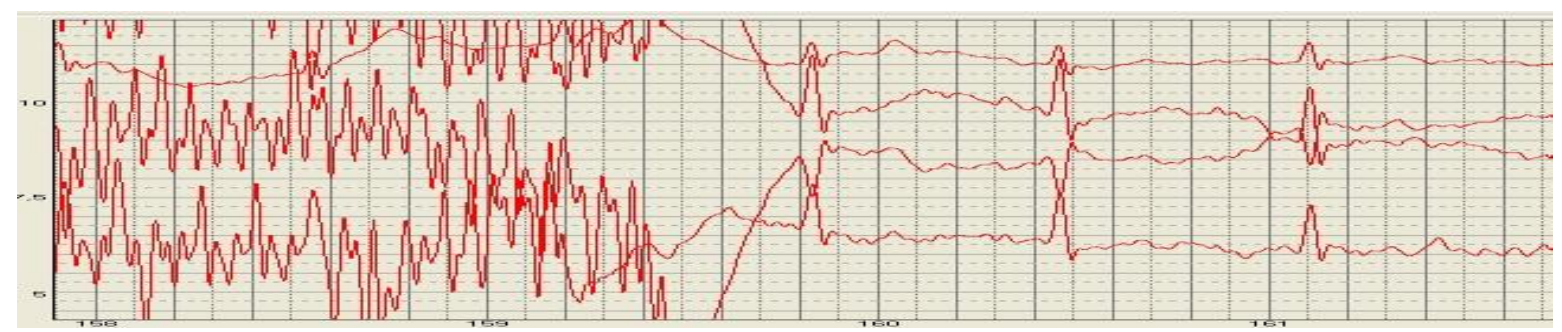

Figure 11. ECG with electrode connection to the subject's skin

Another difference of this recorder from the standard one is the calculation of "autonomous" leads. To obtain leads close to standard, it is necessary to perform mathematical transformations in the program of the receiving computer. These transformations are not difficult and are not the subject of this study. Figure 12 shows 4 ECG channels recorded through a layer of clothing using 4 large textile electrodes and a compensating electrode connected to the human skin. ECG elements on all leads are well distinguishable. 


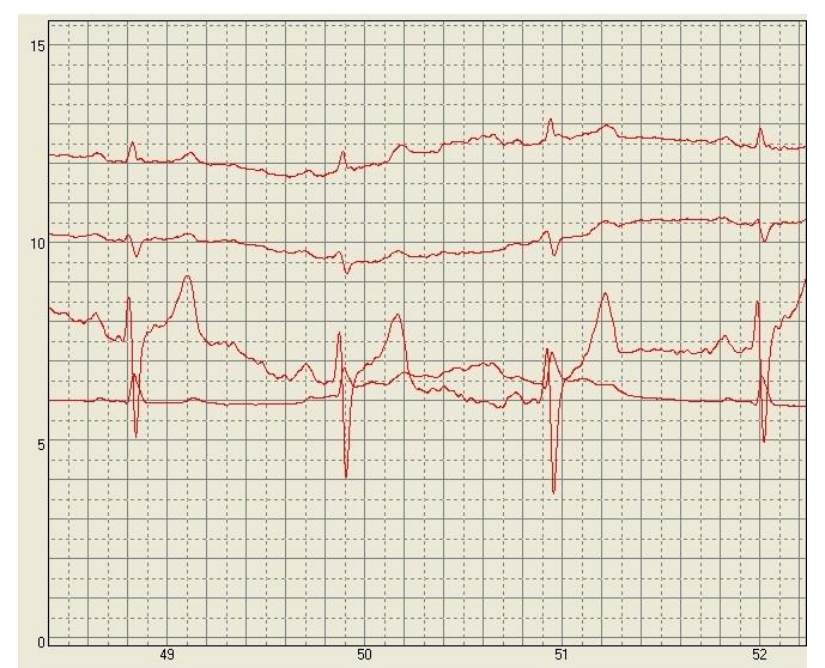

Figure 12. 4 ECG channels recorded through a layer of clothing using 4 textile electrodes of a large area and a compensating electrode connected to the human skin

\section{Discussion}

Thus, as experiments have shown, the combination of autonomous registration of each of the ECG channels using non-inverted inputs of operational amplifiers and the connection of a compensating electrode to the inverse input of the recorder amplifier with a device for "picking up" ECG signals by textile electrodes of a large area is the most suitable option for recording and ECG for continuous monitoring of ECG in personalized medicine and biocybernetic complexes.

For continuous recording of an integral ECG, as one of the vital physiological functions of a person, it is advisable to use recorders with textile electrodes of a large area, placed on bases with a shape memory effect, providing a "pickup" of a signal from a large surface of the human body, as well as with highly sensitive channels for recording electro-biopotentials with a low-impedance compensating input, providing reliable ECG registration with direct contact with human skin only of the compensating electrode and "pseudo-contact" connection of "significant" electrodes.

The quality of registration of an integral ECG using the developed complex of software and hardware allows you to obtain an ECG with a clear identification of the RR intervals, the PQ interval duration, the R wave amplitude and the ST segment value, as well as the ability to analyze the respiratory rate and fatigue of a person and a number of other parameters, characterizing his condition.

\section{Conclusions}

For continuous recording of an integral ECG, as one of the vital physiological functions of a person, it is advisable to use recorders with textile electrodes of a large area, placed on bases with a shape memory effect, providing a "pickup" of a signal from a large surface of the human body, as well as with highly sensitive channels for recording electro-biopotentials with a low-impedance compensating input, providing reliable ECG registration with direct contact with human skin only of the compensating electrode and "pseudo-contact" connection of "significant" electrodes.

The quality of registration of an integral ECG using the developed complex of software and hardware allows you to obtain an ECG with a clear identification of the RR intervals, the PQ interval duration, the R wave amplitude and the ST segment value, as well as the ability to analyze the respiratory rate and fatigue of a person and a number of other parameters, characterizing his condition.

\section{References}

1. Kodkin V.L. Khafizova A. About Non-Contact ECG Recording Devices DOI: 10.26717/BJSTR.2020.24.004047

2. https://www.ti.com/product/ADS1293 
3. Bokeriya O.L. Kodkin V Khafizova A.S.Dynamic electrogardiography for creating innovative methods of the diagnostics of the cardiovascular system DOI: 10.14529/hsm180505

4. Kodkin V Khafizova A.S. A.E. Batueva Analysis of functional abilities in athletes using continious ECG registration under various loads DOI: 10.14529/hsm180408

5. Smirnov A.S., Erlikh V.V., Kodkin V.L., Keller A.V., Epishev V.V. Non-contact ECG monitoring. Progress in Biomedical Optics and Imaging. Proceedings of SPIE. - 2016

6. Kodkin V.L., Yakovleva G.V., Smirnov A.S. Continuous Control Systems for Non-contact ECG. Progress in Biomedical Optics and Imaging. Proceedings of SPIE. - 2017

7. Kodkin V.L. Developing a system for continuous control of the functional status based on recording of electrical potentials and acoustic signals. International Conference on Advances in Biomedicine and Biomedical Engineering 6th International Conference on Biotechnology and Bioengineering ICABBE \& 6th ICBB Offenburg Germany September 26-28, 2017

8. Kodkin V.L., Khafizova A.S. Utility model patent No 176791 «Appliance of human ECG registration for continuous control»

9. Kodkin V.L. (Dubel A.M., Epishev V.V., Smirnov A.S., Erlikh V.V.), Keller A.V., Dorokhov S.A. Utility model patent No 163596 «Appliance for non-contact human electrocardiogram registration»

10. Kodkin V.L. Utility model patent No 130851 «System of automatic control for a patient's physiological state»

11 Gusev M., Poposka L., Spasevski G. Noninvasive Glucose Measurement Using Machine Learning and Neural Network Methods and Correlation with Heart Rate Variability January January 2020 Journal of Sensors ２020(1):1-13 Follow journal DOI:10.1155/2020/9628281

12 http://genphys.phys.msu.ru/rus/diploma/diploma2016/Hapkin.pdf

13. http://www.radiant.su/files/images/plessey/pl_kit_1_2013.pdf

14 https://www.rlocman.ru/review/article.html?di=462751

15 http://www.russianelectronics.ru/leader-r/review/2193/doc/57874/ 